OPEN ACCESS

Edited by:

Cecilia A. Silva-Valenzuela, Centro de Estudios Científicos, Chile

Reviewed by:

Xu Jia,

Chengde Medical College, China

Thomas Jové,

INSERM U1092 Anti-Infectieux supports moléculaires des résistances et innovations thérapeutiques (INSERM), France

*Correspondence: Demeng Tan demengtan@gmail.com

Bijie $\mathrm{Hu}$

hu.bijie@zs-hospital.sh.cn

${ }^{t}$ These authors have contributed equally to this work

Specialty section:

This article was submitted to

Clinical Microbiology,

a section of the journal

Frontiers in Cellular and

Infection Microbiology

Received: 10 October 2021 Accepted: 11 November 2021 Published: 03 December 2021

Citation:

Li N, Zeng Y, Bao R, Zhu T, Tan D and Hu B (2021) Isolation and Characterization of Novel Phages Targeting Pathogenic Klebsiella pneumoniae. Front. Cell. Infect. Microbiol. 11:792305. doi: 10.3389/fcimb.2021.792305

\section{Isolation and Characterization of Novel Phages Targeting Pathogenic Klebsiella pneumoniae}

\author{
$\mathrm{Na} \mathrm{Li}^{1+}$, Yigang Zeng ${ }^{2 \dagger}$, Rong Bao ${ }^{1}$, Tongyu $\mathrm{Zhu}^{1}$, Demeng $\mathrm{Tan}^{2 *}$ and Bijie $\mathrm{Hu}^{1 *}$ \\ ${ }^{1}$ Zhongshan Hospital, Fudan University, Shanghai, China, ${ }^{2}$ Shanghai Public Health Clinical Center, Fudan University, Shanghai, China
}

Klebsiella pneumoniae is a dominant cause of community-acquired and nosocomial infections, specifically among immunocompromised individuals. The increasing occurrence of multidrug-resistant (MDR) isolates has significantly impacted the effectiveness of antimicrobial agents. As antibiotic resistance is becoming increasingly prevalent worldwide, the use of bacteriophages to treat pathogenic bacterial infections has recently gained attention. Elucidating the details of phage-bacteria interactions will provide insights into phage biology and the better development of phage therapy. In this study, a total of $22 \mathrm{~K}$. pneumoniae isolates were assessed for their genetic and phenotypic relatedness by multilocus sequence typing (MLST), endonuclease S1 nuclease pulsed-field gel electrophoresis (S1-PFGE), and in vitro antibiotic susceptibility testing. In addition, the beta-lactamase gene (bla $\mathrm{KPC}_{\mathrm{C}}$ ) was characterized to determine the spread and outbreak of $K$. pneumoniae carbapenemase (KPC)-producing enterobacterial pathogens. Using these ST11 carbapenem-resistant $K$. pneumoniae isolates, three phages (NL_ZS_1, NL_ZS_2, and NL_ZS_3) from the family of Podoviridae were isolated and characterized to evaluate the application of lytic phages against the MDR K. pneumoniae isolates. In vitro inhibition assays with three phages and $K$. pneumoniae strain ZS15 demonstrated the strong lytic potential of the phages, however, followed by the rapid growth of phage-resistant and phage-sensitive mutants, suggesting several anti-phage mechanisms had developed in the host populations. Together, this data adds more comprehensive knowledge to known phage biology and further emphasizes their complexity and future challenges to overcome prior to using phages for controlling this important MDR bacterium.

Keywords: bacteriophage, phage therapy, Klebsiella pneumoniae, phage-host interactions, multidrug resistance

\section{INTRODUCTION}

The ongoing explosion of the antimicrobial resistance crisis has been accelerated by the overuse and misuse of antibiotics, causing the emergence and increased prevalence of multidrug-resistant (MDR) bacteria (Ventola, 2015). Klebsiella pneumoniae, a Gram-negative Enterobacterales, can be found in the environment and has been associated with community-acquired infections (Pendleton et al., 2013). The increasingly common mechanism of carbapenem resistance in $K$. pneumoniae is mediated by extendedspectrum $\beta$-lactamases (ESBLs), which are able to break the $\beta$-lactam structure in the most commonly used antibiotics (Pitout et al., 1997). Thus, infections of KPC-producing bacteria are consistently associated with 
poor clinical outcomes and represent a significant and continued threat to the healthcare system. As a result, there is a strong need to find alternative clinical treatments to conventional antimicrobials.

Bacteriophages (phages for short) are the most common and diverse biological entities in the biosphere, playing an important role in promoting bacterial diversity, structuring community composition, and being a key driver in nutrient turnover (Suttle, 2005). Lytic phages infect and lyse bacterial hosts and are an attractive alternative towards combating the fast development of antibiotic-resistant $K$. pneumoniae (Sulakvelidze et al., 2001; Hung et al., 2011). The use of phages has gained interest over the past years; and phages that are specific for MDR bacterial pathogens, including Pseudomonas aeruginosa, Enterococcus spp., Mycobacterium abscessus, Staphylococcus aureus, Acinetobacter baumannii, and Escherichia coli, have been identified and used to treat bacterial infections (Chibani-Chennoufi et al., 2004b; Capparelli et al., 2007; McVay et al., 2007; Letkiewicz et al., 2009; Schooley et al., 2017; Abd El-Aziz et al., 2019; Dedrick et al., 2019; Cano et al., 2020). These studies demonstrated that lytic phages can be harnessed to prevent and eradicate pathogenic bacteria, and in some cases, alleviate current medical challenges posed by antibacterial resistance and thus further reduce mortalities. While previous applications of phage therapy have shown promising results, the technology is still in a stage of development due to rapid evolution dynamics between phages and their hosts (Chibani-Chennoufi et al., 2004a). It is, therefore, crucial to characterize both phage and host communities prior to any intended phage applications.

Indeed, phage therapy is complicated by the phenotypic and genotypic complexity of both phage and its bacterial host, consisting of strains with different ranges of susceptibility (Ceyssens et al., 2011; Silva-Valenzuela and Camilli, 2019). In order to make phage therapy viable and wildly shared, it is desirable to find a well-characterized collection of phages that, in combination, cover a broad range of hosts. One proposed solution is a combination of two or more phages with different host ranges in a cocktail to delay the appearance of phageresistant mutants (Tanji et al., 2004).

In this study, we analyzed the biological features of a collection of 22 clinical K. pneumoniae isolates, specifically on the characteristics of multi-locus sequence typing (MLST), plasmid, and antibiotic susceptibility patterns. Using these $K$. pneumoniae isolates, we isolated and characterized three phages native to the hospital sewage. Details of phage host range, genome, morphological characters, and phage-host interactions were established to assess the application of phage therapy against these carbapenem-resistant isolates. Together, our study provides important information regarding phage therapy as an environmentally friendly practice to treat and prevent this important human pathogen.

\section{MATERIALS AND METHODS}

\section{Bacterial Strains and Growth Conditions}

Twenty-two nonduplicate bacterial isolates of $K$. pneumoniae were collected from Zhongshan Hospital (Shanghai, China) and identified with MALDI-TOF Mass Spectrometry (Bruker, USA) from June 2019 - May 2020. Isolates were routinely grown in either lysogeny broth (LB) containing per liter: tryptophan $10 \mathrm{~g}$, $\mathrm{NaCl} 10 \mathrm{~g}$, and Yeast extract $5 \mathrm{~g}$ with aeration, or, on LB agar (15 g agar per liter) plates at $37^{\circ} \mathrm{C}$. The beta-lactamase gene (bla $a_{\mathrm{KPC}}$ ) was detected by PCR Master Mix (Thermo, CA, USA) with forward primer 5'-TGTAAGTTACCGCGCTGAGG-3' and reverse primer 5'-CCAGACGACGGCATAGTCAT-3'. All strains were stored at $-80^{\circ} \mathrm{C}$ in $\mathrm{LB}$ broth with $25 \%$ glycerol (vol/vol) and sub-cultivated before each use. Klebsiella pneumoniae strain Kp36, E. coli DH5 $\alpha$, and Salmonella enterica S7 were used as reference strains, and phage 117 was included as well, which belongs to the Caudovirales order, Podoviridae family (Tan et al., 2020).

\section{Multi-Locus Sequence Typing (MLST) and Antibiotic Susceptibility of Bacterial Isolates}

MLST with 7 housekeeping genes ( $r p o B, g a p A, m d h, p g i, p h o E$, $\inf B$, and $\operatorname{ton} B$ ) were performed to characterize these 22 isolates, according to the protocol available on the MLST Pasteur website (https://bigsdb.pasteur.fr/klebsiella/klebsiella.html) (Diancourt et al., 2005). The purified MLST amplicons were sent for Sanger sequencing, followed by comparison with those available on the MLST database to determine the sequence type (ST). The VITEK2 compact automated system (bioMérieux, Lyon, France) was used to determine antibiotic susceptibility profiles according to the manufacturer's instructions. The susceptibility breakpoints were interpreted with reference to the latest documents from the Clinical and Laboratory Standards Institute (CLSI).

\section{S1-Pulsed Field Gel Electrophoresis (S1-PFGE)}

Klebsiella with plasmid-mediated carbapenem resistance poses significant challenges in clinical practice. To characterize the plasmid content in each strain, S1 nuclease digestion analyzed with PFGE was performed using a CHEF Mapper XA apparatus (Bio-Rad Laboratories, Hercules, CA). Briefly, the adjusted bacterial suspension mixed in a $1 \%$ agarose gel was digested with a proteinase $\mathrm{K}$ solution for $2 \mathrm{~h}$ at $54^{\circ} \mathrm{C}$, then further treated with S1 nuclease. The electrophoresis conditions used in this study were as follows: initial switch time, $3 \mathrm{~s}$; a final switch time, $36 \mathrm{~s}$; gradient, $6 \mathrm{~V} / \mathrm{cm}$; angle, 120 ; temperature, $14^{\circ} \mathrm{C}$; and time, $18.5 \mathrm{~h}$. After the electrophoresis run was complete, the gel was stained with $1 \mathrm{mg} \mathrm{mL}^{-1}$ ethidium bromide and visualized with the ChemiDoc XRS system (Bio-Rad Laboratories, CA, USA). Genetic similarities were measured using the Dice coefficient and the clustering was carried out by the unweighted-pair group method with arithmetic averages (UPGMA) in BioNumerics (Applied Maths, St-Martens-Latern, Belgium).

\section{Isolation and Proliferation of Phage}

Phages were isolated using an enrichment protocol as described previously (Tan et al., 2014). Briefly, infiltrated hospital sewage was mixed with the same volume of $2 \times$ LB broth and incubated 
at $37^{\circ} \mathrm{C}$ for $6 \mathrm{~h}$ to stimulate proliferation of total abundances of specific phages targeting the $K$. pneumoniae isolates above. Aliquots of enrichment samples were centrifuged $(12,000 \times g$, $10 \mathrm{~min}, 4^{\circ} \mathrm{C}$ ) and sterilized with $0.1 \%$ chloroform. The presence of $K$. pneumoniae-phages in the hospital sewage samples was detected by double-layer agar assays with different fold dilutions of the cell-free spent supernatant spent from the enrichment assay. In order to obtain individual phages, single plaques were picked and purified three times with their corresponding hosts. Phage stocks were enriched and titrated using double-layer agar assays as described previously and stored at $4^{\circ} \mathrm{C}$ before use (Kropinski et al., 2009).

\section{Phage-Host Range Assay}

The host ranges of obtained phages and phage 117 were determined against 22 clinical isolates along with a well-characterized reference K. pneumoniae strain Kp36, E. coli $\mathrm{DH} 5 \alpha$, and Salmonella enterica S7 by standard spot assays (Tan et al., 2020). Aliquots of $2 \mu \mathrm{L}$ diluted phage lysate $\left(\sim 10^{5} \mathrm{PFU} \mathrm{ml} \mathrm{m}^{-1}\right)$ were spotted on lawns containing $4 \mathrm{~mL}$ top agar mixed with $300 \mu \mathrm{L}$ mid-log phase bacteria. After incubating at $37^{\circ} \mathrm{C}$ for $12 \mathrm{~h}$, the spots were assessed by the turbidity of plaques and characterized as strong infection (clear), weak infection (turbid), or no inhibition (no plaque). The experiment was undertaken as independent duplicates.

\section{Susceptibility of Strain ZS15 to Phage Infection and Isolation of Phage- Resistant Mutants}

Overnight bacterial cultures were 1,000-fold diluted into LB broth and grown to an exponential phase with an OD600 at 0.3 and infected with single phages or phage cocktail (NL_ZS_1, NL_ZS_2, and NL_ZS_3) at a multiplicity of infection (MOI) of 0.01 . The suspension was incubated at $37^{\circ} \mathrm{C}$ with aeration and samples were harvested at $1-\mathrm{h}$ intervals. The phage lysis efficiency was determined by optical density measurements at $\mathrm{OD}_{600} \mathrm{~nm}$. The experiment was done in triplicate along with parallel control cultures without phages. At time point 8-h, single phage lysates were streaked onto LB agar plates and individual colonies were subsequentially purified to determine phage susceptibility by spot test assays as described above.

\section{Phage DNA Extraction and Genomic Analysis}

For the extraction of phage DNA, $2 \mathrm{~mL}$ aliquots of phage lysate with a titer of approximately $10^{9} \mathrm{PFU} \mathrm{mL}^{-1}$ was digested with DNase I (Thermo, CA, USA) and RNase A (Promega, USA) to remove any residual bacterial genomic DNA and RNA according to the manufacturer's protocol. After digesting for $1.5 \mathrm{~h}$ at $37^{\circ} \mathrm{C}$, EDTA (Thermo, CA, USA) was added to a final concentration of $20 \mathrm{mM}$ to inactivate DNase I and RNase A. Total phage DNA was extracted using the Qiagen DNeasy Blood and Tissue kit (Qiagen, CA, USA) following the manufacturer's instructions. Genomic DNA was sent to Sangon Biotech (Shanghai, China) and sequenced using an Illumina HiSeq sequencer.

The raw sequence containing the phage genome was quality controlled and trimmed with FastQC 0.11.2 (https://www. bioinformatics.babraham.ac.uk/projects/fastqc/) and
Trimmomatic 0.36 (http://www.usadellab.org $/ \mathrm{cms} /$ ?page= trimmomatic). The trimmed reads were assembled into a single raw contig via SPAdes 3.5.0. (http://cab.spbu.ru/software/spades/). Genes were predicted and annotated using Rapid Annotation using Subsystem Technology (RAST, http://rast.nmpdr.org/). Phage virulence factor was examined using Virulence Factor Database (VFDB, http://www.mgc.ac.cn/VFs/). Phylogenetic relationship between the genome of phage NL_ZS_1, NL_ZS_2, and NL_ZS_3 and the genomes of related phages were analyzed using CLC Main Workbench (CLC, Qiagen). To construct the phylogenetic tree of phage terminase large subunits, the reference amino acid sequences of were collected from the NCBI database. The terminase large subunits were aligned, and neighbor-joining trees were created using Jukes-Cantor model with a bootstrap analysis of 1000 replicates.

\section{Bacteriophage Morphology by Transmission Electron Microscopy (TEM)}

Based on the genomic differences, phages were selected for examination by TEM. Phage stocks were back-diluted in a SM buffer (50 mM Tris-Cl, pH 7.5, 99 mM NaCl, 8 mM MgSO $4,0.01 \%$ gelatin) to a titer of $10^{8} \mathrm{PFU} \mathrm{mL} \mathrm{m}^{-1}$. Diluted phage lysates were then placed on the surface of Formvar carbon-coated grids (SigmaAldrich, St. Louis, MO, USA) for 5 min to allow adsorption. Grids were stained with $2 \%$ sodium phosphotungstate for 2 min prior to being washed in a drop of distilled water and air-dried before being examined using a JEM-2100 microscope operated at $80 \mathrm{kV}$.

\section{RESULTS}

\section{Isolation and Characterization of K. pneumoniae Strains}

The emergence of carbapenem-resistant K. pneumoniae (CRKP) has become one major burden of healthcare-associated infection due to the dispersion of bacteria carrying the $b l a_{\mathrm{KPC}}$ gene (Bush and Bradford, 2016). KPC was first found in carbapenem-resistant $K$. pneumoniae strains in 2001 in North America, and now KPCproducing strains can be frequently isolated from nosocomial samples. It is, therefore, essential to determine and characterize the nosocomial spread of KPC clones and their corresponding phenotype and genotype. Together, 22 carbapenem-resistant $K$. pneumoniae strains were recovered from various clinical specimens (Zhongshan Hospital, Shanghai, China). According to the $K$. pneumoniae MLST database, all the 22 clinical isolates belonged to ST11 (allelic profile 3-3-1-1-1-1-4), known as the predominant clone of KPC-producing K. pneumoniae in China (Table 1). Despite more than 100 different sequence types (STs) being reported worldwide, the mass dissemination of carbapenemaseproducing K. pneumoniae has been restricted largely to ST258, ST11, ST340, and ST512 (Pitout et al., 2015).

\section{B-Lactamase Content and Plasmid Characterization}

In addition, the $b l a_{\mathrm{KPC}}$ gene was detected in 19 out of 22 isolates, indicating the prevalence of $\beta$-lactamase nosocomial pathogens (Table 1). The $b l a_{\mathrm{KPC}}$ gene can be disseminated between species 
by horizontal (lateral) gene transfer (HGT) via various means, such as plasmids, transposons, prophages, and integrative conjugative elements (ICEs), playing an important effect in generating genotypic heterogeneity and phenotypic variation among bacterial populations (Hardiman et al., 2016; Botelho and Schulenburg, 2020). For instance, transfer of interspecies KPC can occur through the dissemination of mobile genetic elements between strains of S. aureus (Goren et al., 2010).

Since pathogenicity and antimicrobial resistance genes are mainly coded by conjugative plasmids and integrative conjugative elements (ICEs, also known as conjugative transposons), in order to identify the subtypes of K. pneumoniae ST11, pulsed-field gel electrophoresis with S1 nuclease (S1-PFGE) were utilized to resolve the prevalence of plasmid fingerprints of all the 22 isolates, particularly large ones. All groups harbored one to two plasmids ranging from $20 \mathrm{Kbp}$ and 250 Kbp, which were clustered into 6 primary clades (A, B, C, D, E, and F) (Figure 1). Clade A and B were the most common types, while the other clades (C, D, E, and F) only accounted for $31.8 \%$. Thus, it appears that S1-PFGE is a highly discriminative molecular typing method capable of effectively discriminating ST11 clones into several different subtypes, indicating much heterogeneity among these KPCproducing isolates.

\section{Preliminary Antibiotic Susceptibility Testing of $K$. pneumoniae Isolate}

Individual antimicrobial data can be found in Table 1. For ticarcillin/clavulanic acid, piperacillin/tazobactam, cefepime, aztreonam, ciprofloxacin, and levofloxacin, drug resistance was detected in all strains. However, low percentages of resistance to doxycycline, minocycline, tigecycline, colistin, and trimethoprim/ sulfamethoxazole were also observed for most strains. Of these, 17 out of the 22 isolates become resistant to both imipenem and meropenem with a MIC of $\geq 16 \mu \mathrm{g} \mathrm{mL}$, while the other 5 were both sensitive to imipenem and meropenem, except for strain ZS18 which was resistant to imipenem but intermediate to meropenem. For these 5 sensitive isolates, 2 of them were confirmed by PCR and sequencing to have the $b l a_{\mathrm{KPC}}$ gene. Interestingly, strain ZS4 harboring bla $a_{\mathrm{KPC}}$ was susceptible to imipenem and meropenem, perhaps indicating the resistance is associated with changes in gene expression at the mRNA levels (Senchyna et al., 2019).

\section{Isolation and Characterization of Phages}

Three phages (NL_ZS_1, NL_ZS_2, and NL_ZS_3) were isolated from the hospital sewage using the enrichment protocol. The host range of the phages (NL_ZS_1, NL_ZS_2, NL_ZS_3, and phage 117) and their lytic efficiency against all the 23 Klebsiella isolates and two Enterobacterales strains (E. coli $\mathrm{DH} 5 \alpha$, and $S$. enterica S7) was assessed using the spot test assay. According to the host range profile, the phages were able to infect all the $23 \mathrm{~K}$. pneumoniae bacterial isolates, but none of the phages were able to produce plaques on the strain E. coli $\mathrm{DH} 5 \alpha$ or S. enterica S7 (Figure 2), indicating all the phages used in this study failed to lysis relevant Enterobacterales species. The host range patterns and lytic efficiency showed two unique profiles. Based on these profiles, phages were divided into two major groups. Specifically, phage NL_ZS_3 displayed the broadest host range, while phage NL_ZS_1, phage NL_ZS_2, and phage 117 showed a relatively

TABLE 1 | MLST types and antibiotic susceptibility profiles of the 22 K. pneumoniae isolates.

\begin{tabular}{|c|c|c|c|c|c|c|c|c|c|c|c|c|c|c|c|c|c|c|c|}
\hline \multicolumn{20}{|c|}{ Minimum Inhibitory Concentration $(\mu \mathrm{g} / \mathrm{mL})$} \\
\hline Strain & MLST & $b / a_{\mathrm{KPC}}$ & TIM & TZP & CAZ & CPZ & FEP & ATM & IMP & MEM & AMK & тОВ & CIP & LVX & DOX & MIN & TGC & CST & SXT \\
\hline ZS1 & ST11 & Yes & $\geq 128$ & $\geq 128$ & $\geq 64$ & $\geq 64$ & $\geq 32$ & $\geq 64$ & $\geq 16$ & $\geq 16$ & $\geq 64$ & $\geq 16$ & $\geq 4$ & $\geq 8$ & 2 & 4 & $\leq 0.5$ & $\leq 0.5$ & $\leq 20$ \\
\hline ZS2 & ST11 & Yes & $\geq 128$ & $\geq 128$ & $\geq 64$ & 32 & $\geq 32$ & $\geq 64$ & $\geq 16$ & $\geq 16$ & 4 & 8 & $\geq 4$ & $\geq 8$ & 2 & 8 & 1 & $\geq 16$ & $\leq 20$ \\
\hline ZS3 & ST11 & No & $\geq 128$ & $\geq 128$ & $\geq 64$ & $\geq 64$ & $\geq 32$ & $\geq 64$ & 1 & 1 & $\geq 64$ & $\geq 16$ & $\geq 4$ & $\geq 8$ & 4 & $\geq 16$ & 2 & $\leq 0.5$ & $\leq 20$ \\
\hline ZS4 & ST11 & Yes & $\geq 128$ & $\geq 128$ & 32 & $\geq 64$ & $\geq 32$ & $\geq 64$ & 0.5 & $\leq 0.25$ & $\geq 64$ & $\geq 16$ & $\geq 4$ & $\geq 8$ & 2 & 8 & 1 & $\leq 0.5$ & $\leq 20$ \\
\hline ZS5 & ST11 & Yes & $\geq 128$ & $\geq 128$ & $\geq 64$ & $\geq 64$ & $\geq 32$ & $\geq 64$ & $\geq 16$ & $\geq 16$ & 4 & $\leq 1$ & $\geq 4$ & $\geq 8$ & 8 & $\geq 16$ & 4 & $\leq 0.5$ & $\leq 20$ \\
\hline ZS6 & ST11 & Yes & $\geq 128$ & $\geq 128$ & $\geq 64$ & $\geq 64$ & $\geq 32$ & $\geq 64$ & $\geq 16$ & $\geq 16$ & $\geq 64$ & $\geq 16$ & $\geq 4$ & $\geq 8$ & 1 & 2 & $\leq 0.5$ & $\leq 0.5$ & $\leq 20$ \\
\hline ZS7 & ST11 & Yes & $\geq 128$ & $\geq 128$ & $\geq 64$ & $\geq 64$ & $\geq 32$ & $\geq 64$ & $\geq 16$ & $\geq 16$ & 4 & $\leq 1$ & $\geq 4$ & $\geq 8$ & $\geq 16$ & $\geq 16$ & $\geq 8$ & $\leq 0.5$ & $\leq 20$ \\
\hline ZS8 & ST11 & Yes & $\geq 128$ & $\geq 128$ & $\geq 64$ & $\geq 64$ & $\geq 32$ & $\geq 64$ & $\geq 16$ & $\geq 16$ & $\geq 64$ & $\geq 16$ & $\geq 4$ & $\geq 8$ & 4 & 8 & 2 & $\leq 0.5$ & $\leq 20$ \\
\hline ZS9 & ST11 & Yes & $\geq 128$ & $\geq 128$ & $\geq 64$ & $\geq 64$ & $\geq 32$ & $\geq 64$ & $\geq 16$ & $\geq 16$ & $\geq 64$ & $\geq 16$ & $\geq 4$ & $\geq 8$ & 4 & 8 & 2 & $\leq 0.5$ & $\leq 20$ \\
\hline ZS10 & ST11 & Yes & $\geq 128$ & $\geq 128$ & $\geq 64$ & $\geq 64$ & $\geq 32$ & $\geq 64$ & $\geq 16$ & $\geq 16$ & $\geq 64$ & $\geq 16$ & $\geq 4$ & $\geq 8$ & $\geq 16$ & $\geq 16$ & 4 & 4 & $\geq 320$ \\
\hline ZS11 & ST11 & Yes & $\geq 128$ & $\geq 128$ & $\geq 64$ & $\geq 64$ & $\geq 32$ & $\geq 64$ & $\geq 16$ & $\geq 16$ & $\geq 64$ & $\geq 16$ & $\geq 4$ & $\geq 8$ & 2 & 8 & 2 & $\leq 0.5$ & $\leq 20$ \\
\hline ZS12 & ST11 & Yes & $\geq 128$ & $\geq 128$ & $\geq 64$ & $\geq 64$ & $\geq 32$ & $\geq 64$ & $\geq 16$ & $\geq 16$ & $\geq 64$ & $\geq 16$ & $\geq 4$ & $\geq 8$ & 2 & 8 & 1 & $\leq 0.5$ & $\leq 20$ \\
\hline ZS13 & ST11 & Yes & $\geq 128$ & $\geq 128$ & $\geq 64$ & $\geq 64$ & $\geq 32$ & $\geq 64$ & $\geq 16$ & $\geq 16$ & $\geq 64$ & $\geq 16$ & $\geq 4$ & $\geq 8$ & 2 & 8 & $\leq 0.5$ & $\leq 0.5$ & $\leq 20$ \\
\hline ZS14 & ST11 & No & $\geq 128$ & $\geq 128$ & 32 & $\geq 64$ & $\geq 32$ & $\geq 64$ & 1 & 0.5 & $\geq 64$ & $\geq 16$ & $\geq 4$ & $\geq 8$ & 2 & 8 & $\leq 0.5$ & $\leq 0.5$ & $\leq 20$ \\
\hline ZS15 & ST11 & Yes & $\geq 128$ & $\geq 128$ & $\geq 64$ & $\geq 64$ & $\geq 32$ & $\geq 64$ & $\geq 16$ & $\geq 16$ & $\geq 64$ & $\geq 16$ & $\geq 4$ & $\geq 8$ & 2 & 8 & 8 & $\leq 0.5$ & $\leq 20$ \\
\hline ZS16 & ST11 & Yes & $\geq 128$ & $\geq 128$ & $\geq 64$ & $\geq 64$ & $\geq 32$ & $\geq 64$ & $\geq 16$ & $\geq 16$ & $\geq 64$ & $\geq 16$ & $\geq 4$ & $\geq 8$ & 4 & $\geq 16$ & 2 & $\geq 16$ & $\leq 20$ \\
\hline ZS17 & ST11 & Yes & $\geq 128$ & $\geq 128$ & $\geq 64$ & $\geq 64$ & $\geq 32$ & $\geq 64$ & $\geq 16$ & $\geq 16$ & $\geq 64$ & $\geq 16$ & $\geq 4$ & $\geq 8$ & 4 & 8 & 1 & $\leq 0.5$ & $\leq 20$ \\
\hline ZS18 & ST11 & Yes & $\geq 128$ & $\geq 128$ & $\geq 64$ & 32 & $\geq 32$ & $\geq 64$ & $\geq 16$ & 8 & 8 & $\leq 1$ & $\geq 4$ & $\geq 8$ & $\leq 0.5$ & 2 & $\leq 0.5$ & $\leq 0.5$ & $\leq 20$ \\
\hline ZS19 & ST11 & No & $\geq 128$ & $\geq 128$ & 8 & $\geq 64$ & $\geq 32$ & $\geq 64$ & $\leq 0.25$ & $\leq 0.25$ & $\geq 64$ & $\geq 16$ & $\geq 4$ & $\geq 8$ & 2 & 4 & $\leq 0.5$ & $\leq 0.5$ & $\leq 20$ \\
\hline ZS20 & ST11 & Yes & $\geq 128$ & $\geq 128$ & $\geq 64$ & $\geq 64$ & $\geq 32$ & $\geq 64$ & $\geq 16$ & $\geq 16$ & $\geq 64$ & $\geq 16$ & $\geq 4$ & $\geq 8$ & 1 & 4 & $\leq 0.5$ & $\leq 0.5$ & $\leq 20$ \\
\hline ZS21 & ST11 & Yes & $\geq 128$ & $\geq 128$ & $\geq 64$ & $\geq 64$ & $\geq 32$ & $\geq 64$ & $\geq 16$ & $\geq 16$ & $\geq 64$ & $\geq 16$ & $\geq 4$ & $\geq 8$ & 1 & 4 & $\leq 0.5$ & $\leq 0.5$ & $\leq 20$ \\
\hline ZS22 & ST11 & Yes & $\geq 128$ & $\geq 128$ & $\geq 64$ & $\geq 64$ & $\geq 32$ & $\geq 64$ & $\geq 16$ & $\geq 16$ & $\geq 64$ & $\geq 16$ & $\geq 4$ & $\geq 8$ & 1 & 2 & $\leq 0.5$ & $\leq 0.5$ & $\leq 20$ \\
\hline
\end{tabular}

Antibiotic abbreviations: TIM, ticarcillin/clavulanic acid; TZP, piperacillin/tazobactam; CAZ, ceftazidime; CPZ, cefoperazone; FEP, cefepime; ATM, aztreonam; IPM, imipenem; MEM, meropenem; AMK, amikacin; TOB, tobramycin; CIP, ciprofloxacin; LVX, levofloxacin; DOX, doxycycline; MIN, minocycline; TGC, tigecycline; CST, colistin; SXT, Trimethoprim/Sulfamethoxazole. 


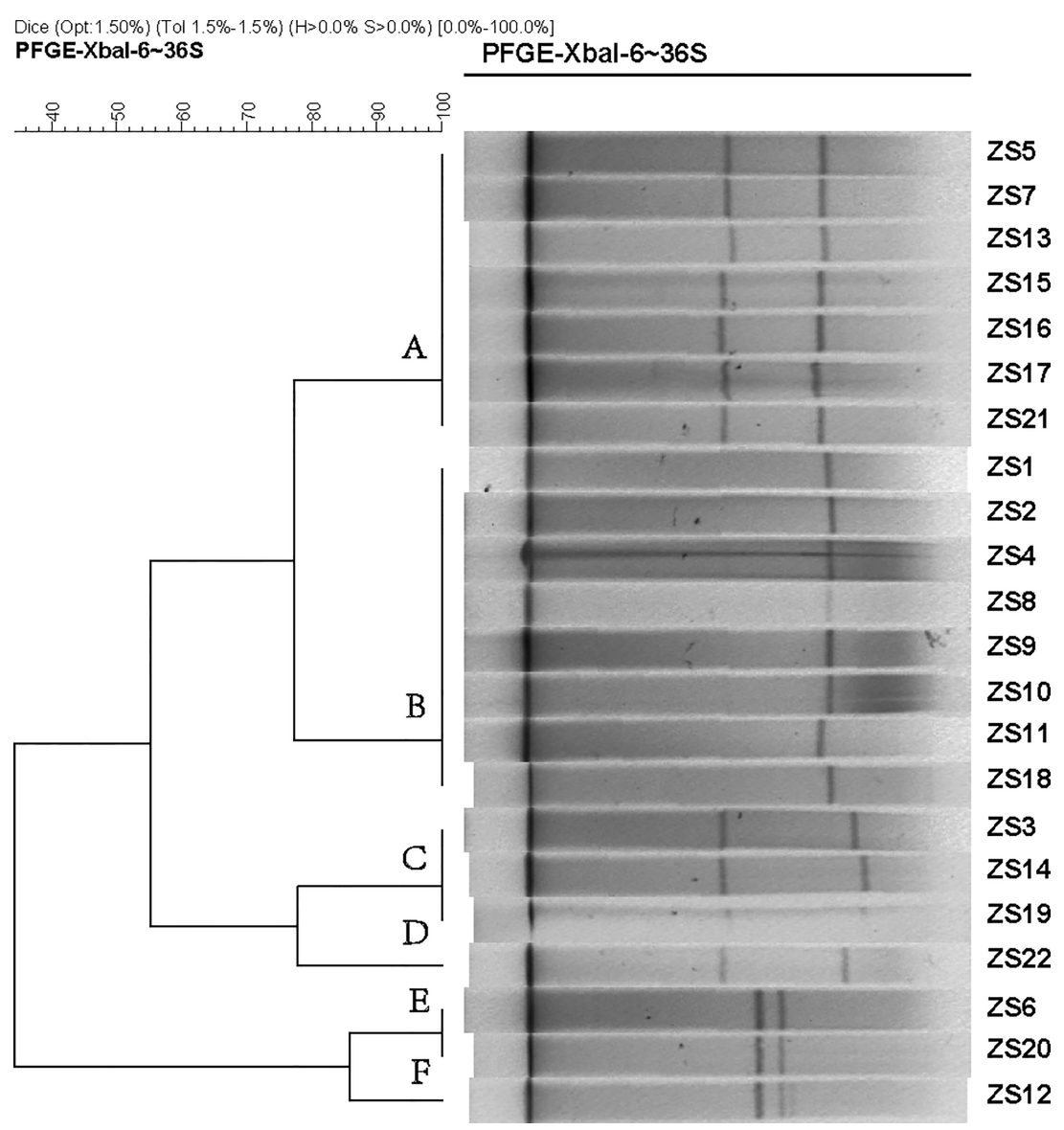

FIGURE 1 | Dendrogram generated by BioNumerics software (Version7.1, Applied Maths, St-Martens-Latem, Belgium) showing cluster results of molecular epidemiology S1-PFGE analysis of 22 K. pneumoniae isolates. Band-based similarity coefficients were calculated using Dice coefficient and unweighted pair group average method algorithm (UPGMA). Salmonella enterica serotype Braenderup strain H9812 was digested with Xbal and used as a molecular size marker. The electrophoresis conditions were as follows: initial switch time, $3 \mathrm{~s}$; a final switch time, $36 \mathrm{~s}$; gradient, $6 \mathrm{~V} / \mathrm{cm}$; angle, 120 ; temperature, $14^{\circ} \mathrm{C}$ and time, $18.5 \mathrm{~h}$.

narrow host range, infecting only $\sim 50 \%$ of the tested $K$. pneumoniae strains (Figure 2). The host range differences between the phages observed in this study are likely due to the different phage characterization, i.e., efficiency of adsorption and lytic efficiency in its potential hosts.

\section{Morphology and Genome Sequence of Phages}

All phage lysates formed transparent plaques on strain ZS15, which are considered as an indicator of strong lysis. Plaque morphologies of all phages presented a zone of clear lysis on the host strain ZS15, while a halo zone was detected on the plaques of both phages NL_ZS_1 and NL_ZS_2, indicating the production of polysaccharide depolymerase (Figure 3 ). Depolymerase activity is usually encoded by their tail fibers or tail spikes protein on the baseplate. Since depolymerases target and destroy cell wall structures, they have been suggested as potential antimicrobial agents to conventional antibiotics.

Based on TEM analysis, all the phages belong to the family Podoviridae of Caudovirales order, having short, non-contractile tails ( 11 nm in length) and icosahedral heads ( 61 nm diameter) (Figure 3 and Table 2). In addition, total genomic DNA was extracted from each of the three phages, and their genomes were sequenced by Illumina MiSeq. The assembly sizes of these three phages ranged from $38 \mathrm{~kb}$ to $40 \mathrm{~kb}$, having a $\mathrm{G}+\mathrm{C}$ content ranging between $50 \%$ to $53 \%$, with 45 to 49 predicted protein-coding genes (Table 2). All phage genomes (GenBank accession: phage NL_ZS_1, MT813140; phage NL_ZS_2, MT813141; and phage NL_ZS_3, MT813142) were submitted to RAST (Rapid Annotation using Subsystem Technology) and further annotated by the available pipeline. No identifiable virulence genes, antibiotic resistance genes, or genes involved in lysogenic cycles were detected in the genomes. Therefore, it appears that those phages isolated in this study can satisfy the safety assessment based on their complete genome sequences. Progressive multiple genome alignments were analyzed using Easyfig software (https://mjsull.github.io/Easyfig/) (Figure 4) to determine the relatedness of three $K$. pneumoniae phages. A considerable relation was shown between phage NL_ZS_1 and phage NL_ZS_2, while phage NL_ZS_2 displays a relatively low 


\begin{tabular}{|c|l|l|l|l|}
\hline \multirow{2}{*}{ Strain } & \multicolumn{5}{|c|}{ Phage } \\
\hline & NL_ZS_1 & NL_ZS_2 & NL_ZS_3 & D117 \\
\hline ZS1 & & & & \\
\hline ZS2 & & & & \\
\hline ZS3 & & & & \\
\hline ZS4 & & & & \\
\hline ZS5 & & & & \\
\hline ZS6 & & & & \\
\hline ZS7 & & & & \\
\hline ZS8 & & & & \\
\hline ZS9 & & & & \\
\hline ZS10 & & & & \\
\hline ZS11 & & & & \\
\hline ZS12 & & & & \\
\hline ZS13 & & & & \\
\hline ZS14 & & & & \\
\hline ZS15 & & & & \\
\hline ZS16 & & & & \\
\hline ZS17 & & & & \\
\hline ZS18 & & & & \\
\hline ZS20 & & & & \\
\hline ZS21 & & & & \\
\hline ZS22 & & & & \\
\hline Kp36 & & & & \\
\hline DH5 2 & & & & \\
\hline S7 & & & & \\
\hline
\end{tabular}

FIGURE 2 | Phage-host range assay of the 4 phages (columns) against the 22 K. pneumoniae strains and the reference strain Kp36, DH5 $\alpha$, and S7 (rows). Klebsiella pneumoniae phages displayed broad and narrow host ranges. Black, grey and white indicate infection, weak infection and no plaque formation, respectively.

homologous region with phage NL_ZS_3. Likely, phylogenetic analysis of the large subunit of the terminase of phage NL_ZS_1, NL_ZS_2, and NL_ZS_3 reveals their close relation to large terminase subunits of other phages, specifically, phage NL_ZS_1 and phage NL_ZS_2 display high similarities to Klebsiella phage K11, BIS33, and KpV289, while phage NL_ZS_3 was closely related to Salmonella phage phiSG-JL2 (Figure 5). Overall, these results showed high relation to the phage-host range pattern, demonstrating phage susceptibility correlates with phage core genome, such as genes involved in the phage tail fibers.

\section{Phage Growth Characteristics in LB Broth}

To examine the efficiency of phage infection, $K$. pneumoniae ZS15 was infected in liquid cultures with single phages and phage cocktail (NL_ZS_1, NL_ZS_2, and NL_ZS_3) at a MOI of $~ 0.01$, respectively, and their inhibition was assessed by bacterial growth following the measurement of the optical density of the bacterial cultures with and without phages. An inhibitory effect of phage NL_ZS_1 and phage NL_ZS_2 was observed in the in vitro inhibition assay with a drastic decline in optical density on the initial control of the host population for $1.5 \mathrm{~h}$ followed by 

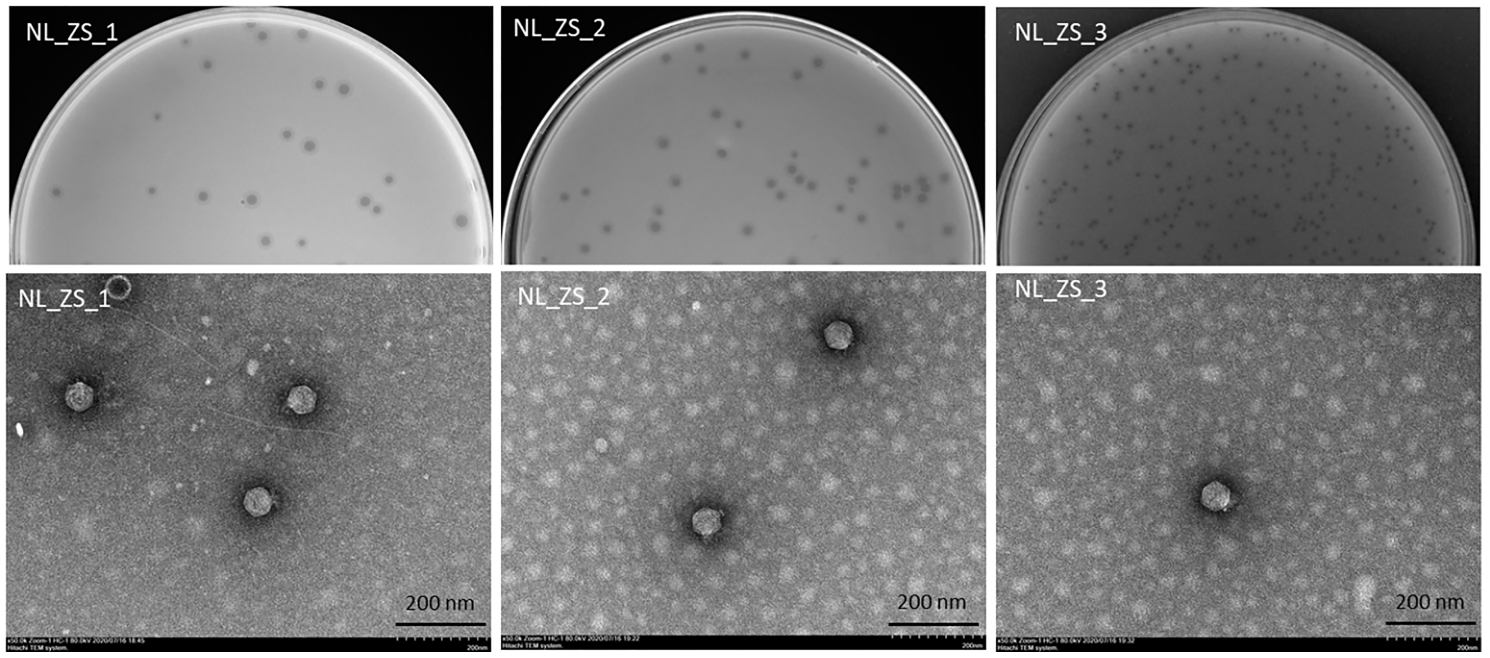

FIGURE 3 | Plaque images (up) and their corresponding TEM micrographs (below). Phages were stained with $2 \%$ sodium phosphotungstate and were found to have short small podophage morphology. Scale bar, $200 \mathrm{~nm}$.

regrowth of the resistant population (Figure 6). Likely, a similar trend in the phage-amended cultures of ZS15+ NL_ZS_3 was observed in the first $5 \mathrm{~h}$, however, it was followed by a less pronounced decrease and finally stabilized after $6 \mathrm{~h}$ (Figure 6). To assess the applicability of the phage cocktail, we performed the same growth curve inhibition analysis on the strain ZS15. As expected, the phage cocktail showed a stronger distribution of growth inhibition than that of phage NL_ZS_3, indicating the strain ZS15 can be suppressed for longer by the phage cocktail combining three different phages. After $8 \mathrm{~h}$, the OD600 $\mathrm{nm}$ of the phage-amended cultures of group 1 (ZS15+phage NL_ZS_1 and ZS15+phage NL_ZS_2), group 2 (ZS15+phage NL_ZS_3), and group 3 (phage cocktail) stabilized at 1.95, 1.96, 0.09, and 0.05, corresponding to $52 \%$ to $98 \%$ of the final OD in the control group, respectively (Figure 6). Together, these results demonstrate that phage cocktail were more effective in reducing bacterial densities compared with the single phage, thus prolonging the usable time of the phage cocktail, and increasing the efficiency in phage application.

Despite K. pneumoniae ZS15 displaying a strong susceptibility to infection by all three phages, phage NL_ZS_3 was better at suppressing resistance to host strain ZS15 over phage NL_ZS_1 and NL_ZS_2. Bacteria are known to rapidly develop phage resistance when subjected to predation by lytic phages in a laboratory medium (Labrie et al., 2010). By screening phage resistance mutants with spot test, we attempted to better understand the inhibition differences among each of the phageamended cultures. To do this, phage insensitive mutants were isolated from phage lysate cultures during the incubation for the analysis of changes in their susceptibility upon phage selection. Based on the results, we observed that phage-amended cultures (ZS15+phage NL_ZS_1, ZS15+phage NL_ZS_2, and ZS15+phage NL_ZS_3) were dominated by resistant non-mucoid clones after $8 \mathrm{~h}$ of incubation, indicating conversion of mucoid $\mathrm{K}$. pneumoniae strain ZS15 to non-mucoid variants by consequence of selective pressure operated by phages (Table 3). However, in the ZS15 +phage NL_ZS_3 cultures, heterogeneity in phage susceptibility was observed (Table 3). Out of $30 \mathrm{~K}$. pneumoniae ZS15 isolates, 2 ( $6.7 \%)$ were fully sensitive to phage NL_ZS_3 and displayed the hypermucoviscosity (HV) phenotype as the parental strain ZS15, and partially sensitive (i.e., reduced phage susceptibly when compared to the parental strain) subpopulations ( 50\%) were isolated as well and did not revert back to the wild-type phenotype despite three rounds of purification. Interestingly, $43.3 \%$ were fully resistant to phage NL_ZS_3, however, a small fraction ( 30\%) of phage NL_ZS_3 resistant mutants reverted back to the parental phenotype after three rounds of purification, suggesting different anti-phage mechanisms were allowing coexistence of phage sensitive mutants and that their lytic phages could exist between bacterial host ZS15 and phage NL_ZS_3. We do not know, however, the dynamics of phenotype heterogeneity over time or how long such persistence can last before heritage mutations

TABLE 2 | Morphological and genomic characterization of phages isolated from the local hospital sewage.

\begin{tabular}{|c|c|c|c|c|c|c|c|c|c|c|c|}
\hline Phage & Family & Host & $\begin{array}{l}\text { Head diam } \\
(\mathrm{nm})\end{array}$ & $\begin{array}{l}\text { Head length } \\
\quad(\mathrm{nm})\end{array}$ & $\begin{array}{l}\text { Tail diam } \\
\quad(\mathrm{nm})\end{array}$ & $\begin{array}{c}\text { Tail length } \\
\text { (nm) }\end{array}$ & $\begin{array}{c}\text { Genome size } \\
\text { (bp) }\end{array}$ & $\begin{array}{c}\text { GC } \\
\%\end{array}$ & $\begin{array}{l}\text { Genome } \\
\text { type }\end{array}$ & $\begin{array}{l}\text { Predicted } \\
\text { genes }\end{array}$ & Source \\
\hline NL_ZS_1 & Podoviridae & ZS15 & 63 & 58 & 13 & 14 & 40,428 & 53 & dsDNA & 45 & Sewage \\
\hline NL_ZS_2 & Podoviridae & ZS15 & 58 & 63 & 13 & 9 & 40,222 & 52.8 & dsDNA & 49 & Sewage \\
\hline NL_ZS_3 & Podoviridae & ZS15 & 63 & 63 & 13 & 11 & 38,741 & 50.7 & dsDNA & 46 & Sewage \\
\hline
\end{tabular}




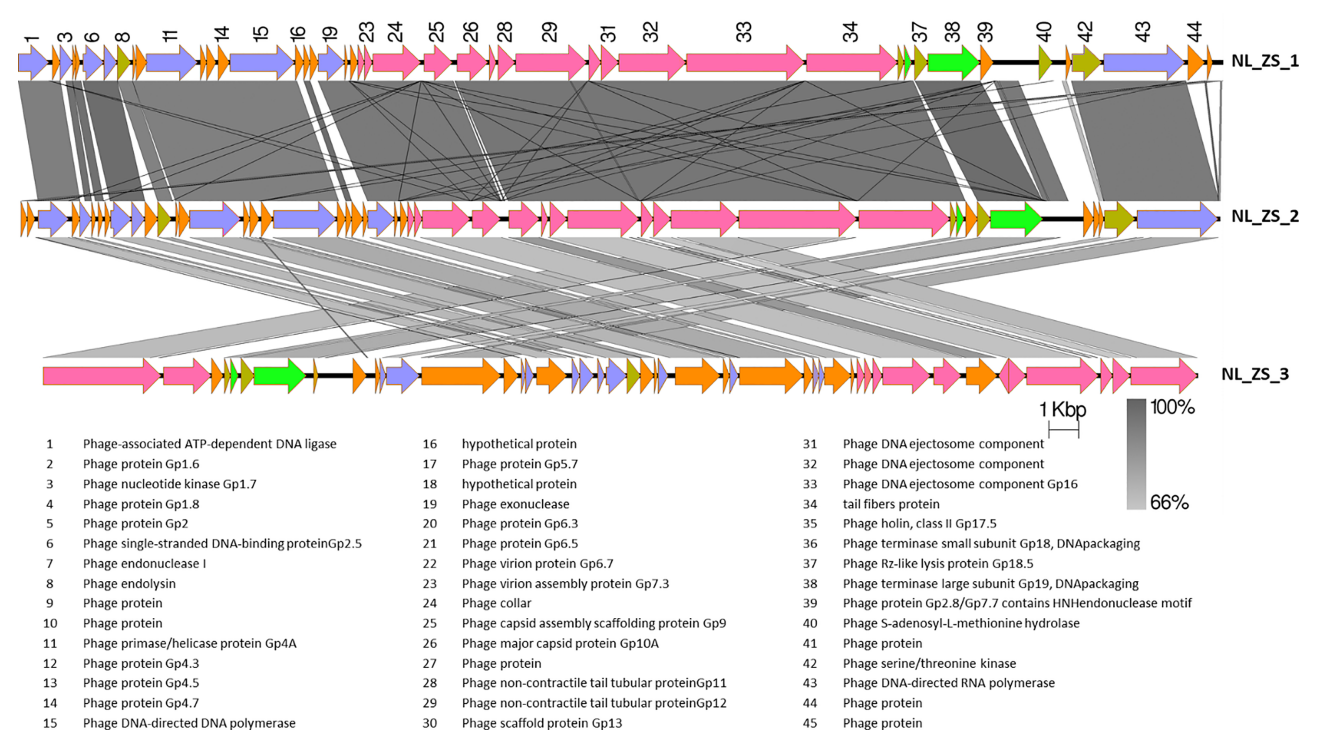

FIGURE 4 | The comparison of the whole genome sequences among three $K$. pneumoniae phages (NL_ZS_1, NL_ZS_2, and NL_ZS_3) with Easyfig using the BLASTN algorithm. Grey lines connect regions indicates nucleotide identity $>66 \%$, and are gradient-colored according to their similarity; darker shade of grey represents higher identify. The designation of the open reading frames of NL_ZS_1 is shown on the top. Conserved ORFs are color-coded with blue arrows representing the DNA replication module, yellow arrows representing host cell lysis module, pink reparenting the capsid and tail morphogenesis module, green arrows representing the DNA packaging module, and borrow arrows represent genes with no known function.

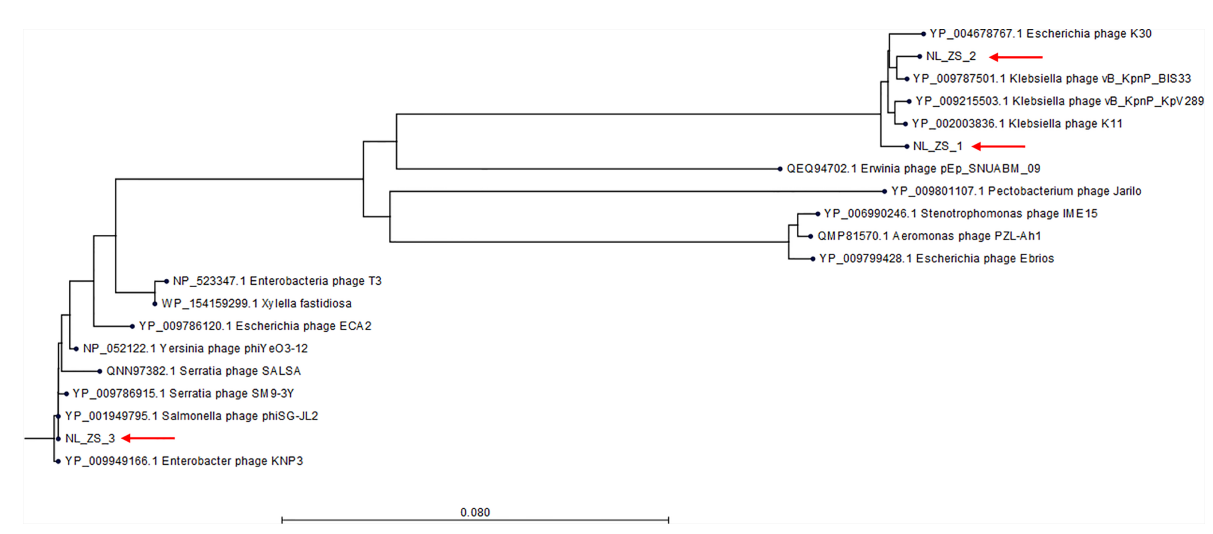

FIGURE 5 | Phylogenetic tree was constructed based on the amino acid sequence of the larger terminase subunit of phages NL_ZS_1, NL_ZS_2, and NL_ZS_3 and phages sharing homology sequence identity retrieved from GenBank (https://www.ncbi.nlm.nih.gov/). The evolutionary history of 20 larger terminase subunit amino acid sequences were aligned and inferred using Neighbor Joining method and 1000 bootstrap replicates (CLC Genomics Workbench 8).

dominate the entire population, since phage susceptibility was only measured at $8 \mathrm{~h}$,

\section{Anti-Phage Defense-Mediated Altered Sensitivity to Tigecycline}

Previous studies have shown that evolutionary phage-host interactions affect phage resistance and antibiotic susceptibility both positively and negatively, and are highly genotypedependent in bacterial populations (Comeau et al., 2007; Chan et al., 2016; Burmeister et al., 2020). We started to question whether phage-resistant mutants changed their antibiotic susceptibilities via antagonistic coevolution between hosts and parasites in LB broth. To investigate this further, we performed Vitek 2 assays for antimicrobial susceptibility, testing phage NL_ZS_3-resistant mutants (ZS15- NL_ZS_3R) and their parental phage susceptible strains (ZS15). Interestingly, for tigecycline susceptibility testing of carbapenem-resistant $K$. pneumoniae ZS15 strain, we noticed that strain ZS15 evolved resistance to phage NL_ZS_3, while simultaneously becoming sensitive to tigecycline with the minimum inhibitory 


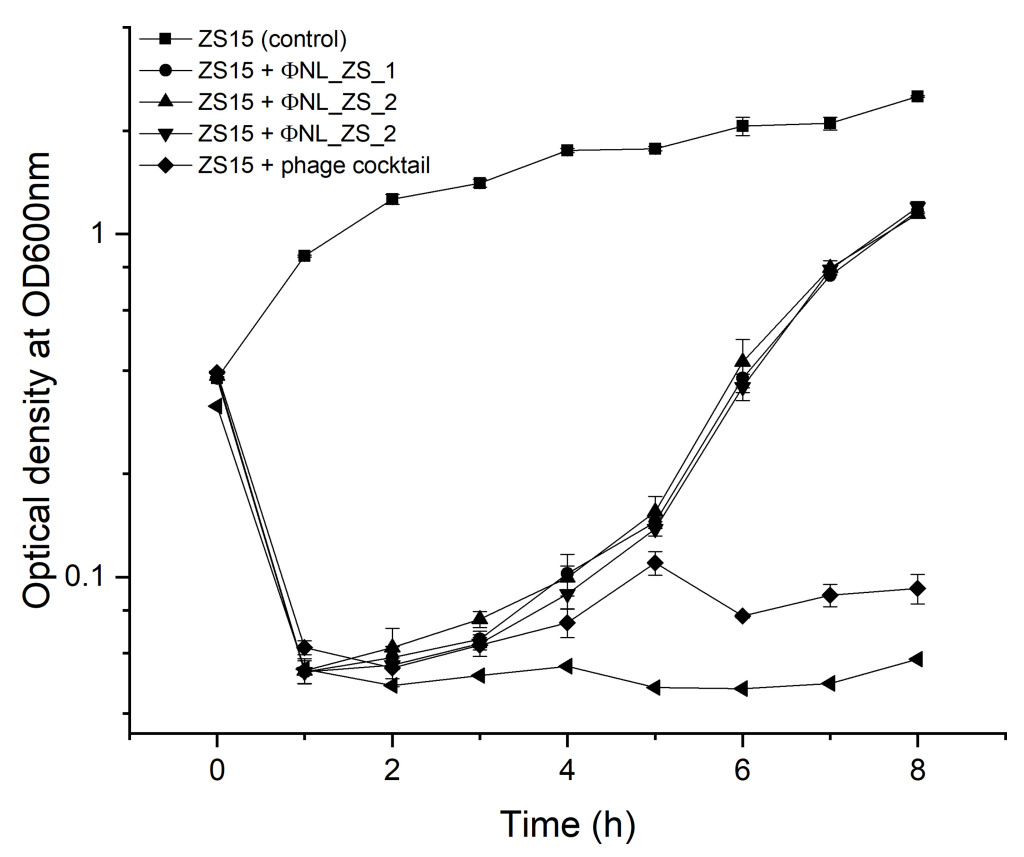

FIGURE 6 | Inhibition assay of single phages and phage cocktail (NL_ZS_1, NL_ZS_2, and NL_ZS_3) in the treatment at a MOI of 0.1 and control without phage in cultures of $K$. pneumoniae ZS15. Optical density (OD600) was measured at 1-h intervals over an 8-h incubation period. Error bars represent the standard deviations from all experiments, carried out in triplicate.

concentration shifting from $8 \mu \mathrm{g} \mathrm{mL}^{-1}$ to $2 \mu \mathrm{g} \mathrm{mL} \mathrm{m}^{-1}$. Unfortunately, we were unable to identify potential genes related to phage selection and antibiotic sensitivity during the interactions between strain ZS15 and phage NL_ZS_3. Overall, these results indicate that an evolutionary trade-off between phage resistance and antibiotic resistance. Further, comparative genomic sequence analyses are needed to elucidate the underlying mechanisms by which restoration of antibiotic susceptibility occurs.

\section{DISCUSSION}

Klebsiella pneumoniae is a common nosocomial pathogen that causes difficult-to-treat infections worldwide. The prevalence and distribution of bla $a_{K P C}$ gene in $K$. pneumoniae is increasing, making carbapenem resistance isolates difficult to treat due to the lack of effective and safe alternative options. Successful application of phage therapy in treating K. pneumoniae caused infections requires a detailed understanding of the phenotypic and genotypic diversity of $K$. pneumoniae pathogens and phages. In this study, we characterized 22 carbapenem-resistant $K$. pneumoniae strains and 3 lytic phages. Further, K. pneumoniae strain ZS15 was selected for more detailed studies of phage lytic potential and phage-host interactions.

\section{Diversity of $K$. pneumoniae ST11 Isolates}

The clonal relationship between $K$. pneumoniae strains examined in the present study belonged to ST11 irrespective of time or place of isolation. ST11 is among the most important clinical dominant in Asia and South America and has been reported in different regions worldwide since its emergence (Qi et al., 2011). In 2018, a fatal outbreak of ST11 carbapenem-resistant hypervirulent $K$. pneumoniae in a Chinese hospital has caused high mortality and morbidity (Gu et al., 2018; Yao et al., 2018). Likewise, ST258, single-locus variants of ST11 (the tonB allele distinguishes the two

TABLE 3 | Phage susceptibility properties of $K$. pneumoniae ZS15 isolates obtained from cultures enriched with the phages.

\begin{tabular}{lccc}
\hline & Percentage of phage susceptibility properties of $\boldsymbol{K}$. pneumoniae ZS15 isolates (\%) & Partially sensitive & Sensitive \\
\hline Phage & Resistant & 0 & 0 \\
\hline NL_ZS_1 & $100 \%$ & 0 & 0 \\
NL_ZS_2 & $100 \%$ & $50 \%$ & $6.7 \%$ \\
NL_ZS_3 & $43.3 \%$ & & 0 \\
\hline
\end{tabular}

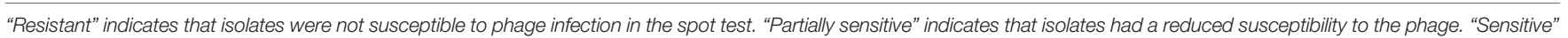
indicates that isolates were fully sensitive to the phage. 
sequence types), has emerged as an important cause of hospital death in the United States and other countries, and is largely responsible for the global spread of KPC (Toth et al., 2010). However, the molecular linage between ST11 and ST258 largely remains unknown. Recent comparative genomic analyses showed ST258 is a hybrid with $\sim 80 \%$ of the chromosome homology to ST11 strains, while the remaining $20 \%$ display high similarity to the ST442 isolates (Chen et al., 2014). Despite MLST does not provide sufficient discrimination for the 22 pathogenic isolates or resolve differences across spatial and temporal sac ales, understanding the molecular evolutionary history of bacterial isolates is an important step towards the development of diagnostic and potential therapeutic strategies to combat infections caused by multidrug-resistant K. pneumoniae.

The antibiotic susceptibility profile presents a worrying trend of antimicrobial resistance, as carbapenem resistance was seen among all Klebsiella isolates. Treatment options for infections with carbapenem non-susceptible $K$. pneumoniae are very limited, leaving colistin as the last treatment option to fight against these infections. Out of 22 strains, 2 isolates (strain ZS2 and ZS16) display resistance to colistin. Currently, there is little understanding of molecular mechanisms underlying antibiotic resistance and its impact on virulence remains unclear, despite $\sim 86 \%$ of strains being confirmed to be carbapenemase gene positive. A recent sequence of complete $K$. pneumoniae genomes showed that genes coding for resistance to antibiotics or virulence factors is located in the plasmid (David et al., 2020). Moreover, the spread of these resistant plasmids into the normal gut flora poses an even great public health threat. To recapitulate the contents and diversity of the plasmids of genetically related $K$. pneumoniae isolates, it is important to track the spread of its plasmids between each isolate. In this study, S1-PFGE showed four clusters of the CRKp producing strains that corresponded to ST11, representing a high level of genetic diversity. Together, these results provide important insights into relatively narrow phylogenetic and phenotypic groups of bacteria, emphasizing the complexity of the development of alternatives to treat this pathogen. Future work will seek to understand the dissemination of these plasmids and their potentials of converting any K. pneumoniae strains with no existing antimicrobial resistance or virulence determinants into CRKp strain.

\section{Phage Isolation and Diversity}

Generally, phages are widespread and genetically diverse in almost all environments, especially in sewage, known as a fertility source for many pathogens. The high occurrence of $K$. pneumoniae phages in the collected samples ( 72\%) suggested the K. pneumoniae phages are widespread in the local hospital sewage. The phages displayed two distinguished host ranges when tested against the strain collection. Only 1 morphological family, Podoviridae, was represented, and the phages ranged in genome size from $38 \mathrm{~kb}$ to $40 \mathrm{~kb}$. Due to the limited sample numbers, we do not know to what extent these isolated phages represent the true diversity and structure of local hospital sewage phage communities. Nevertheless, it would be interesting to experimentally study the viral metagenomics that is not dependent on cell culture approaches to expand our understanding of phage genomic diversity from the evolutionary perspective and perhaps genetic exchanges in response to the selection pressure from the bacterial host.

Indeed, each phage has its own particular host range, defined as the set of strains permissive for its infection. Some broad host range phages can infect multiple species of bacteria. For instance, phage KVP40 is able to infect Vibrio anguillarum, $V$. parahaemolyticus, $V$. harveyi, and $V$. cholerae (Matsuzaki et al., 1992); bacteriophage $\mathrm{Mu}$ infects several species, including E. coli, Citrobacter freundii, Shigella sonnei, Enterobacter, and Erwinia (Ross et al., 2016). However, host ranges can be limited by diverse phage receptors on the surface of bacteria. In this study, phage NL_ZS_3 displayed a relatively broad host range, while phage NL_ZS_1, phage NL_ZS_2, phage 117 shared identical relatively narrow host ranges, suggesting that they might use similar phage binding receptors to recognize bacterial phage receptors (olonic acid) in their susceptible hosts. This is interesting as bioinformatic analysis showed that phagebinding receptors of phage NL_ZS_1 and phage NL_ZS_2 were identical based on the amino acid sequence. The possible explanation for host range difference could be phage NL_ZS_3 used a conservative apparatus as its phage receptors, such as outer membrane proteins, lipopolysaccharides, flagella, or pili. Moreover, several copies of genes encoding phage tails or tail fibers were present in the phage NL_ZS_3, which opens the possibility that phage NL_ZS_3 may use a primary receptor to initiate attachment but require a secondary cell surface receptor to irreversibly bind to the bacterial host for injection, permitting more flexibility to develop on a wider range of hosts. For example, the T4 phage uses its long tail fibers to recognize lipopolysaccharide and the outer membrane protein $\mathrm{C}$, present on the bacterial surface, and this reversible attachment allows the phage to move along the surface and find a suitable site to inject its DNA (Tétart et al., 1996; Baslé et al., 2006).

\section{Phage-Host Interactions in Strain ZS15}

Isolation and characterization of bacterial isolates during the LB broth experiments confirmed the rapid development of phage resistance and subsequently dominated the bacterial populations in the phage NL_ZS_1- and phage NL_ZS_2-amend cultures, which is regarded as a significant barrier to the successful application of phage therapy (Koskella and Meaden, 2013). Interestingly, in the current experiment with strain ZS15, phage NL_ZS_ 3 exposures led to the rapid development of phenotypic diversity (i.e., resistant, partially sensitive, or resistant) within a population that enables survival of fluctuating, suggesting different anti-phage mechanisms were allowing coexistence of phage sensitive mutants and their lytic phages. Since phage susceptibility was only measured at $8 \mathrm{~h}$, we do not know the dynamics of phenotype heterogeneity over time or how long such persistence can last before heritage mutations dominate the entire population. It is, therefore, crucial to characterize the phage-host interaction regarding the phenotypic and genetic diversification in bacteria and phages prior to any intended phage applications.

A common form of developing phage resistance for bacterial hosts is associated with loss or altered structure of the receptors to block the potential phage adsorption in order to avoid infection (Labrie et al., 2010). Such changes are directly linked 
to the key step in recognition between phage receptor-binding proteins (RBP) located on tail fibers, and phage receptors encoded on the susceptible bacterial host cell surface (Bertozzi Silva et al., 2016). In general, the fitness costs resulting from receptor mutation (i.e., motility, nutrient transport, or antimicrobial susceptibility) make the mutants unable to compete with phage-susceptible strains in both environmental samples and infection models, even in the presence of phage predation (Seed et al., 2012; Sumrall et al., 2019). Understanding the evolutionary implication of phage-receptor interactions will be beneficial for predicting how phages interact with their host, as phage receptor mutation-mediated phenotypic variabilities may result in exopolysaccharide associated fitness cost. Such characterization of phage resistance evolution will be used as a guide for selecting proper phage targeting of specific receptors with a significant clinical application. Our data reveal when phage NL_ZS_3 resistance evolves, the resistant mutant strain of ZS15 showed increased tigecycline sensitivity, despite the mechanism underlying potential is not fully understood.

Tigecycline, known as a tetracycline antibiotic, has shown the greatest antimicrobial activity against CRKP. Genes e.g. tet(B), tet $(\mathrm{A})$, tet $(\mathrm{K})$, tet $(\mathrm{M})$ and tet( $\mathrm{S})$ coding for multidrug efflux pumps are membrane-related proteins and can efflux tetracyclines from the bacteria intracellularly, thus decreasing the MIC and subsequently allowing protein synthesis without affecting cell viability (Linkevicius et al., 2016). Using membrane-related proteins as a surface structure is one of the most common components functioning as a phage receptor. Therefore, it makes sense that phage-resistant defense strategies would confer a fitness cost for bacteria, i.e., becoming more sensitive to certain antibiotics. For instance, in $P$. aeruginosa, phage OMKO1 evolved to become less resistant to antibiotics. Likewise, in E. coli, the TolC protein evolved in a variety of diverse cellular functions, including antibiotic efflux and phage TLS receptor (Chan et al., 2016). However, unlike previous studies, the anti-phage TLS mediated TolC mutation did not influence its role in antibiotic efflux. Broadly, these results reveal that a phage-host arm race can result in different outcomes in terms of antibiotic susceptibilities, highlighting the diversity and complexity of host interactions within bacterial populations. Further, additional knowledge behind phage resistance in bacteria pathogens and the physiological consequences of acquiring phage resistance phenotypes mechanisms can be gained to renew the utility of ineffective antibiotics.

\section{Potential Applications of Phage Therapy}

Successful application of phage therapy requires collaboration between microbiology and preclinical development; however, little is known about their alignment to guide future development. Since the discovery of phages, many have been isolated and identified as potential alternatives to antibiotics with different efficacy profiles. Phage specificity is both a benefit and an obstacle for clinical trials, as phage receptor diversity affects phage-host interaction. When the bacterial population is under phage attack, only a small fraction of cells can survive these selective pressures, and those that survive determine the fate of the population. Therefore, elucidating details of phage-host interactions and how these would influence bacterial physiological heterogeneity is fundamental to the development of phage therapy. In this study, the three highlighted phages and phage cocktail showed promising in vitro effects that perhaps these phages can be tamed for our benefits without negatively affecting their natural balance. The main limitation of this study is that only a small number of bacterial isolates and phages have been investigated. A large-scale study on the interactions between carbapenem-resistant $K$ pneumoniae strains and phages covering a considerable spatial and temporal variability is essential for the successful application of phage-based pathogen control to fill our knowledge gap between in vitro and in vivo models.

\section{DATA AVAILABILITY STATEMENT}

The datasets presented in this study can be found in online repositories. The names of the repository/repositories and accession number(s) can be found in the article/supplementary material.

\section{AUTHOR CONTRIBUTIONS}

Conceptualization, DT and NL. Methodology, NL. Software, RB and YZ. Formal analysis, YZ. Investigation, NL and YZ. Resources, DT. Data curation, NL. Writing-original draft preparation, DT. Writing-review and editing, DT and $\mathrm{BH}$. Supervision, DT and $\mathrm{BH}$. Project administration, DT and $\mathrm{BH}$. Funding acquisition, BH, TZ, and DT. All authors contributed to the article and approved the submitted version.

\section{FUNDING}

This project was supported by the National Natural Science Foundation of China (NSFC) (No. 42006136) Shanghai Municipal Commission of Health (No.20204Y0336), Shanghai Public Health Clinical Center (KY-GW-2020-08), and Shanghai Pujiang Talents Plan Project (No. 2020PJ054) to DT and by the National Natural Science Foundation of China (NSFC) (No.82072325) and Clinical Research Plan of SHDC (No. SHDC2020CR2031B) to BH.

\section{ACKNOWLEDGMENTS}

We thank Yiyuan Zhang, Xiao Wang and Ruoyu Li for technical assistance. We thank all members from the Zhu lab for thoughtful discussions and are grateful to Brett C. Gonzalez for proofreading the manuscript. We also wish to thank the editor and two anonymous reviewers for their constructive comments. 


\section{REFERENCES}

Abd El-Aziz, A. M., Elgaml, A., and Ali, Y. M. (2019). Bacteriophage Therapy Increases Complement-Mediated Lysis of Bacteria and Enhances Bacterial Clearance After Acute Lung Infection With Multidrug-Resistant Pseudomonas Aeruginosa. J. Infect. Dis. 219, 1439-1447. doi: 10.1093/infdis/jiy678

Baslé, A., Rummel, G., Storici, P., Rosenbusch, J. P., and Schirmer, T. (2006). Crystal Structure of Osmoporin OmpC From E coli at 2.0 A. J. Mol. Biol. 362, 933-942. doi: 10.1016/j.jmb.2006.08.002

Bertozzi Silva, J., Storms, Z., and Sauvageau, D. (2016). Host Receptors for Bacteriophage Adsorption. FEMS Microbiol. Lett. 363, fnw002. doi: 10.1093/ femsle/fnw002

Botelho, J., and Schulenburg, H. (2020). The Role of Integrative and Conjugative Elements in Antibiotic Resistance Evolution. Trends Microbiol. 29 (1), 8-18. doi: 10.1016/j.tim.2020.05.011

Burmeister, A. R., Fortier, A., Roush, C., Lessing, A. J., Bender, R. G., Barahman, R., et al. (2020). Pleiotropy Complicates a Trade-Off Between Phage Resistance and Antibiotic Resistance. Proc. Natl. Acad. Sci. U. S. A. 117, 11207-11216. doi: 10.1073/ pnas. 1919888117

Bush, K., and Bradford, P. A. (2016). $\beta$-Lactams and $\beta$-Lactamase Inhibitors: An Overview. Cold Spring Harb. Perspect Med. 6, a025247. doi: 10.1101/ cshperspect.a025247

Cano, E. J., Caflisch, K. M., Bollyky, P. L., Van Belleghem, J. D., Patel, R., Fackler, J., et al. (2020). Phage Therapy for Limb-Threatening Prosthetic Knee Klebsiella Pneumoniae Infection: Case Report and In Vitro Characterization of Anti-Biofilm Activity. Arch. Clin. Infect. Dis. 73 (1), e114-51. doi: 10.1093/ cid/ciaa705

Capparelli, R., Parlato, M., Borriello, G., Salvatore, P., and Iannelli, D. (2007). Experimental Phage Therapy Against Staphylococcus Aureus in Mice. Antimicrob. Agents Chemother. 51, 2765-2773. doi: 10.1128/AAC.01513-06

Ceyssens, P.-J., Glonti, T., Lavigne, R., Chanishvili, N., Kulakov, L., Lashkhi, N., et al. (2011). Phenotypic and Genotypic Variations Within a Single Bacteriophage Species. Virol. J. 8, 1-5. doi: 10.1186/1743-422X-8-134

Chan, B. K., Sistrom, M., Wertz, J. E., Kortright, K. E., Narayan, D., and Turner, P. E. (2016). Phage Selection Restores Antibiotic Sensitivity in MDR Pseudomonas Aeruginosa. Sci. Rep. 6, 26717. doi: 10.1038/srep26717

Chen, L., Mathema, B., Pitout, J. D., Deleo, F. R., and Kreiswirth, B. N. (2014). Epidemic Klebsiella Pneumoniae ST258 Is a Hybrid Strain. mBio 5, e01355e01314. doi: 10.1128/mBio.01355-14

Chibani-Chennoufi, S., Sidoti, J., Bruttin, A., Kutter, E., Sarker, S., and Brussow, H. (2004b). In Vitro and In Vivo Bacteriolytic Activities of Escherichia Coli Phages: Implications for Phage Therapy. Antimicrob. Agents Chemother. 48, 2558-2569. doi: 10.1128/AAC.48.7.2558-2569.2004

Chibani-Chennoufi, S., Bruttin, A., Dillmann, M. L., and Brussow, H. (2004a). Phage-Host Interaction: An Ecological Perspective. J. Bacteriol. 186, 36773686. doi: 10.1128/JB.186.12.3677-3686.2004

Comeau, A. M., Tétart, F., Trojet, S. N., Prere, M.-F., and Krisch, H. (2007). PhageAntibiotic Synergy (PAS): $\beta$-Lactam and Quinolone Antibiotics Stimulate Virulent Phage Growth. PLoS One 2, e799. doi: 10.1371/journal.pone.0000799

David, S., Cohen, V., Reuter, S., Sheppard, A. E., Giani, T., Parkhill, J., et al. (2020). Integrated Chromosomal and Plasmid Sequence Analyses Reveal Diverse Modes of Carbapenemase Gene Spread Among Klebsiella Pneumoniae. Proc. Natl. Acad. Sci. 117, 25043-25054. doi: 10.1073/pnas.2003407117

Dedrick, R. M., Guerrero-Bustamante, C. A., Garlena, R. A., Russell, D. A., Ford, K., Harris, K., et al. (2019). Engineered Bacteriophages for Treatment of a Patient With a Disseminated Drug-Resistant Mycobacterium Abscessus. Nat. Med. 25, 730. doi: 10.1038/s41591-019-0437-z

Diancourt, L., Passet, V., Verhoef, J., Grimont, P. A., and Brisse, S. (2005). Multilocus Sequence Typing of Klebsiella Pneumoniae Nosocomial Isolates. J. Clin. Microbiol. 43, 4178-4182. doi: 10.1128/JCM.43.8.4178-4182.2005

Goren, M. G., Carmeli, Y., Schwaber, M. J., Chmelnitsky, I., Schechner, V., and Navon-Venezia, S. (2010). Transfer of Carbapenem-Resistant Plasmid From Klebsiella Pneumoniae ST258 to Escherichia Coli in Patient. Emerg. Infect. Dis. 16, 1014-1017. doi: 10.3201/eid1606.091671

Gu, D., Dong, N., Zheng, Z., Lin, D., Huang, M., Wang, L., et al. (2018). A Fatal Outbreak of ST11 Carbapenem-Resistant Hypervirulent Klebsiella Pneumoniae in a Chinese Hospital: A Molecular Epidemiological Study. TLancet Infect. Dis. 18, 37-46. doi: 10.1016/S1473-3099(17)30489-9
Hardiman, C. A., Weingarten, R. A., Conlan, S., Khil, P., Dekker, J. P., Mathers, A. J., et al. (2016). Horizontal Transfer of Carbapenemase-Encoding Plasmids and Comparison With Hospital Epidemiology Data. Antimicrob. Agents Chemother. 60, 4910-4919. doi: 10.1128/AAC.00014-16

Hung, C. H., Kuo, C. F., Wang, C. H., Wu, C. M., and Tsao, N. (2011). Experimental Phage Therapy in Treating Klebsiella Pneumoniae-Mediated Liver Abscesses and Bacteremia in Mice. Antimicrob. Agents Chemother. 55, 1358-1365. doi: 10.1128/AAC.01123-10

Koskella, B., and Meaden, S. (2013). Understanding Bacteriophage Specificity in Natural Microbial Communities. Viruses 5, 806-823. doi: 10.3390/v5030806

Kropinski, A. M., Mazzocco, A., Waddell, T. E., Lingohr, E., and Johnson, R. P. (2009). "Enumeration of Bacteriophages by Double Agar Overlay Plaque Assay," in Bacteriophages: Methods and Protocols, Volume 1: Isolation, Characterization, and Interactions. Eds. M. R. J. Clokie and A. M. Kropinski (Totowa, NJ: Humana Press), 69-76.

Labrie, S. J., Samson, J. E., and Moineau, S. (2010). Bacteriophage Resistance Mechanisms. Nat. Rev. Microbiol. 8, 317-327. doi: 10.1038/nrmicro2315

Letkiewicz, S., Międzybrodzki, R., Fortuna, W., Weber-Dąbrowska, B., and Górski, A. (2009). Eradication of Enterococcus Faecalis by Phage Therapy in Chronic Bacterial Prostatitis-Case Report. Folia Microbiol. 54, 457. doi: 10.1007/ s12223-009-0064-z

Linkevicius, M., Sandegren, L., and Andersson, D. I. (2016). Potential of Tetracycline Resistance Proteins To Evolve Tigecycline Resistance. Antimicrob. Agents Chemother. 60, 789-796. doi: 10.1128/AAC.02465-15

Matsuzaki, S., Tanaka, S., Koga, T., and Kawata, T. (1992). A Broad-Host-Range Vibriophage, KVP40, Isolated From Sea Water. Microbiol. Immunol. 36, 9397. doi: $10.1111 / j .1348-0421.1992 . t b 01645 . x$

Mcray, C. S., Velasquez, M., and Fralick, J. A. (2007). Phage Therapy of Pseudomonas Aeruginosa Infection in a Mouse Burn Wound Model. Antimicrob. Agents Chemother. 51, 1934-1938. doi: 10.1128/AAC.01028-06

Pendleton, J. N., Gorman, S. P., and Gilmore, B. F. (2013). Clinical Relevance of the ESKAPE Pathogens. Expert Rev. Anti Infect. Ther. 11, 297-308. doi: 10.1586/eri.13.12

Pitout, J. D., Nordmann, P., and Poirel, L. (2015). Carbapenemase-Producing Klebsiella Pneumoniae, a Key Pathogen Set for Global Nosocomial Dominance. Antimicrob. Agents Chemother. 59, 5873-5884. doi: 10.1128/AAC.01019-15

Pitout, J. D., Sanders, C. C., and Sanders, J. W. E. (1997). Antimicrobial Resistance With Focus on $\beta$-Lactam Resistance in Gram-Negative Bacilli. Am. J. Med. 103, 51-59. doi: 10.1016/S0002-9343(97)00044-2

Qi, Y., Wei, Z., Ji, S., Du, X., Shen, P., and Yu, Y. (2011). ST11, the Dominant Clone of KPC-Producing Klebsiella Pneumoniae in China. J. Antimicrob. Chemother. 66, 307-312. doi: 10.1093/jac/dkq431

Ross, A., Ward, S., and Hyman, P. (2016). More Is Better: Selecting for Broad Host Range Bacteriophages. Front. Microbiol. 7, 1352. doi: 10.3389/fmicb. 2016.01352

Schooley, R. T., Biswas, B., Gill, J. J., Hernandez-Morales, A., Lancaster, J., Lessor, L., et al. (2017). Development and Use of Personalized Bacteriophage-Based Therapeutic Cocktails to Treat a Patient With a Disseminated Resistant Acinetobacter Baumannii Infection. Antimicrob. Agents Chemother. 61, e00954-17 . doi: 10.1128/AAC.00954-17

Seed, K. D., Faruque, S. M., Mekalanos, J. J., Calderwood, S. B., Qadri, F., and Camilli, A. (2012). Phase Variable O Antigen Biosynthetic Genes Control Expression of the Major Protective Antigen and Bacteriophage Receptor in Vibrio Cholerae O1. PloS Pathog. 8, e1002917. doi: 10.1371/journal.ppat.1002917

Senchyna, F., Gaur, R. L., Sandlund, J., Truong, C., Tremintin, G., Kültz, D., et al. (2019). Diversity of Resistance Mechanisms in Carbapenem-Resistant Enterobacteriaceae at a Health Care System in Northern California, From 2013 to 2016. Diagn. Microbiol. Infect. Dis. 93, 250-257. doi: 10.1016/j.diagmicrobio. 2018.10.004

Silva-Valenzuela, C. A., and Camilli, A. (2019). Niche Adaptation Limits Bacteriophage Predation of Vibrio Cholerae in a Nutrient-Poor Aquatic Environment. Proc. Natl. Acad. Sci. U. S. A. 116, 1627-1632. doi: 10.1073/ pnas. 1810138116

Sulakvelidze, A., Alavidze, Z., and Morris, J. G. Jr. (2001). Bacteriophage Therapy. Antimicrob. Agents Chemother. 45, 649-659. doi: 10.1128/AAC.45.3.649659.2001

Sumrall, E. T., Shen, Y., Keller, A. P., Rismondo, J., Pavlou, M., Eugster, M. R., et al. (2019). Phage Resistance at the Cost of Virulence: Listeria Monocytogenes 
Serovar 4b Requires Galactosylated Teichoic Acids for InlB-Mediated Invasion. PLoS Pathog. 15, e1008032. doi: 10.1371/journal.ppat.1008032

Suttle, C. A. (2005). Viruses in the Sea. Nature 437, 356-361. doi: 10.1038/nature04160 Tan, D., Gram, L., and Middelboe, M. (2014). Vibriophages and Their Interactions With the Fish Pathogen Vibrio Anguillarum. Appl. Environ. Microbiol. 80, 3128-3140. doi: 10.1128/AEM.03544-13

Tanji, Y., Shimada, T., Yoichi, M., Miyanaga, K., Hori, K., and Unno, H. (2004). Toward Rational Control of Escherichia Coli O157: H7 by a Phage Cocktail. Appl. Environ. Microbiol. 64, 270-274. doi: 10.1007/s00253-003-1438-9

Tan, D., Zhang, Y., Qin, J., Le, S., Gu, J., Chen, L.-K., et al. (2020). A Frameshift Mutation in wcaJ Associated With Phage Resistance in Klebsiella Pneumoniae. Microorganisms 8, 378. doi: 10.3390/microorganisms8030378

Tétart, F., Repoila, F., Monod, C., and Krisch, H. (1996). Bacteriophage T4 Host Range Is Expanded by Duplications of a Small Domain of the Tail Fiber Adhesin. J. Mol. Biol. 258 (5), 726-731.

Toth, A., Damjanova, I., Puskas, E., Jánvári, L., Farkas, M., Dobák, A., et al. (2010). Emergence of a Colistin-Resistant KPC-2-Producing Klebsiella Pneumoniae ST258 Clone in Hungary. Eur. J. Clin. Microbiol. Infect. Dis. 29, 765-769. doi: 10.1007/s10096-010-0921-3

Ventola, C. L. (2015). The Antibiotic Resistance Crisis: Part 1: Causes and Threats. Pharm. Ther. 40, 277-283.
Yao, H., Qin, S., Chen, S., Shen, J., and Du, X.-D. (2018). Emergence of Carbapenem-Resistant Hypervirulent Klebsiella Pneumoniae. Lancet Infect. Dis. 18, 25. doi: 10.1016/S1473-3099(17)30628-X

Conflict of Interest: The authors declare that the research was conducted in the absence of any commercial or financial relationships that could be construed as a potential conflict of interest.

Publisher's Note: All claims expressed in this article are solely those of the authors and do not necessarily represent those of their affiliated organizations, or those of the publisher, the editors and the reviewers. Any product that may be evaluated in this article, or claim that may be made by its manufacturer, is not guaranteed or endorsed by the publisher.

Copyright $\odot 2021 \mathrm{Li}$, Zeng, Bao, Zhu, Tan and Hu. This is an open-access article distributed under the terms of the Creative Commons Attribution License (CC BY). The use, distribution or reproduction in other forums is permitted, provided the original author(s) and the copyright owner(s) are credited and that the original publication in this journal is cited, in accordance with accepted academic practice. No use, distribution or reproduction is permitted which does not comply with these terms. 